\title{
Transvestic Fetishism
}

National Cancer Institute

\section{Source}

National Cancer Institute. Transvestic Fetishism. NCI Thesaurus. Code C94359.

A disorder characterized by recurrent sexual urges, fantasies, or behaviors in a heterosexual male involving cross-dressing. 